\title{
The Three Color Processes in the Retina of Frog.
}

By

Koiti Motokawa and Kituya Iwama.

(本川弘一) (岩間吉也)

(From the Physiological Laboratory of Prof. K. Motokawa,

Tohoku University, Sendai.)

(Received for publication, April 20, 1949)

The electrical excitability of excised frog eyes following two seconds'. illumination by white or colored light was studied, taking the least perceptible impulses of the optic nerve set up by electrical stimulation of the eye as the index of retinal excitation. The stimulating current was a constant current pulse of one second in duration. Examples of the excitability curves which represent the time course of excitability changes following illumination are illustrated in Fig. 1. The curve for white light shows three distinct humps which may be denoted by $R, G$ and $B$ (see (a) in Fig. 1). The time to the maximum or the crest time of each hump remains unaltered as the intensity of light is varied over a wide range. This finding suggests that in the retina of frog, there are three kinds of processes with different time constants. The three processes are elicited in different proportions according to the wave-length of light just as the trichromatic theory advocates. Fig. 1 (b) represents the excitability curves for red light, which provokes the $\mathbf{R}$ process greatly, and the $G$ process only slightly, but can-

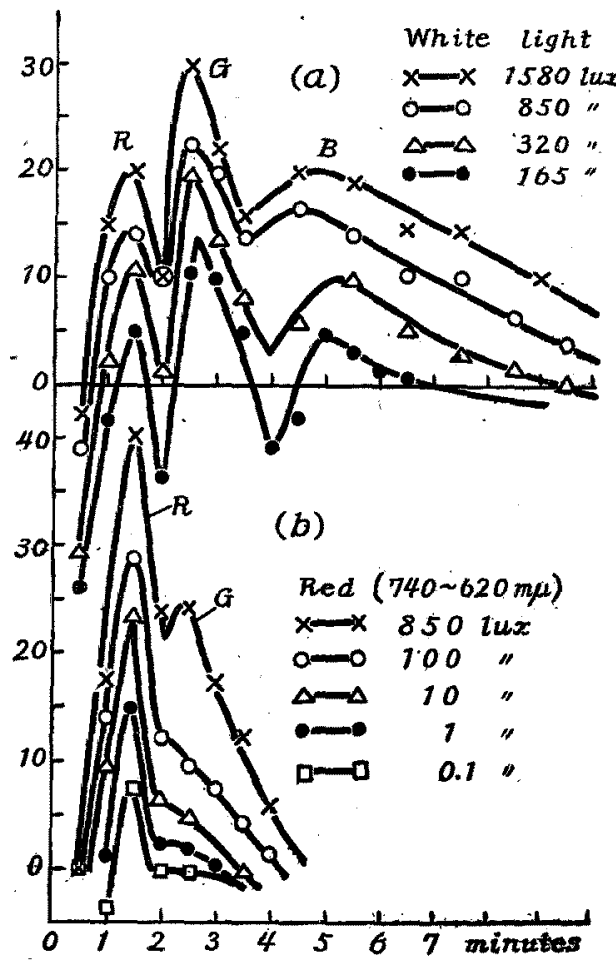

Fig. 1. Ordinate: percentage increase of electrical excitability.

Abscissa: time from the end of illumination to electrical stimulation. not elicit the $B$ process. 